\title{
Effects of skeletal muscle denervation on potency of rocuronium
}

\author{
Wang Hong ${ }^{\mathrm{a}}$, Qi-Sheng Liang, Lan-Ren Cheng ${ }^{\mathrm{a}}$, Xiao-Hong Li ${ }^{\mathrm{a}}$, Fu Wei ${ }^{\mathrm{a}}$, Wen-Tao Dai ${ }^{\mathrm{a}}$, Shi-Tong Li ${ }^{\mathrm{b}}$ \\ a Department of Anesthesiology, the First Affiliated Hospital of Bengbu Medical College, Bengbu, \\ Anhui 233000; ' Department of Anesthesiology, First People's Hospital, School of Medicine, Shanghai \\ Jiaotong University, Shanghai 200030, China
}

\begin{abstract}
Background: Rocuronium is an alternative to succinylcholine for rapid tracheal intubation after major thermal injury and other forms of critical illness that cause denervation changes in skeletal muscle. Rocuronium may decrease the potencies of non-depolarizing muscle relaxants.

Objectives: Examine whether potency of rocuronium changed during the first month after denervation, and investigate the effects of skeletal muscle denervation on potency of rocuronium.

Methods: The denervation mouse model was developed to create denervated individual cells from the flexor digitorum brevis of the hindfoot. The skeletal muscle cells were examined at day 0 in the innervated control and days 1, 4, 7, 14, 21, and 28 in the denervation group. Nicotinic acetylcholine receptors in the cells were activated with 30 M acetylcholine, alone or in combination with various concentrations of rocuronium. Currents were recorded with a whole-cell patch-clamp technique.

Results: Rocuronium reversibly inhibited acetylcholine-activated currents in a dose-dependent fashion at different times after denervation. The inhibition concentration for the half-maximal responses of rocuronium increased 1.2- ( $\mathrm{p}>0.05), 1.8-$, 2.8-, 2.3-, 2.1-, and 1.9-fold ( $<<0.01$ ) at day 1, 4, 7, 14, 21, and 28 after denervation, respectively, compared to that at day 0 after denervation.

Conclusion: Rocuronium dose required to achieve satisfactory clinical effects changed at different durations after skeletal muscle denervation.
\end{abstract}

Keywords: Potency, rocuronium, skeletal muscle denervation, whole-cell patch-clamp technique

After major thermal injury and other forms of critical illness, situations may arise in which rapid onset of paralysis is required, such as rapid-sequence induction of anesthesia or for treatment of laryngospasm. Clinical use of succinylcholine is contraindicated because of lethal hyperkalemia. Rocuronium has a fast onset time, an intermediate duration of action, and no hyperkalemia, and may well be an alternative to succinylcholine for rapid tracheal intubation.

However, thermal injury and other forms of critical illness cause denervation changes in skeletal muscle,

Correspondence to: Shi-Tong Li, Department of Anesthesiology, First People's Hospital, School of Medicine, Shanghai Jiaotong University, Shanghai 200030, China. E-mail: wh201071 @gmail.com which results in decreased potencies of nondepolarizing muscle relaxants (NDMRs) [1, 2]. Skeletal muscle denervation causes up-regulation of nicotinic acetylcholine receptors (nAChRs) and expression de novo of the fetal-type nicotinic acetylcholine receptor ( $\gamma$-AChR) [3-5]. After denervation, the amounts of nAChR and $\gamma$-AChR change with time. Furthermore, the $\mathrm{IC}_{50}$ of dTC correlates positively with nAChRs and AChR $\gamma$-mRNA at different times after denervation [4, 6, 7]. All of these indicate that the rocuronium doses required to achieve satisfactory tracheal intubation may differ at different times after denervation.

In this study, we measured the changes in potency of rocuronium during the first one month after denervation in a mouse model. 


\section{Methods \\ Denervation}

This study was approved by the Animal Care and Use Committee of the Shanghai JiaoTong University School of Medicine.

$\mathrm{Balb} / \mathrm{C}$ mice (35 days old) were anesthetized with pentobarbital, $40 \mathrm{mg} / \mathrm{kg}$ ip. A few millimeter of the right sciatic nerve was excised through a small (3-5 $\mathrm{mm}$ ) incision over the hip. The incision was sutured with a single stitch. Animals were killed at 1, 4, 7, 14, 21 , or 28 days after denervation by pentobarbital anesthesia and cervical dislocation. Animals that had received surgery but not sectioning of the sciatic nerve were used as the innervated controls and examined at day 0 .

\section{Isolation of muscle fibers}

Single skeletal muscle cells from the flexor digitorum brevis (FDB) muscle were obtained from the hindfeet of each mouse. The muscles were incubated for three hours with Dulbecco's modified Eagle's medium (DMEM) (Invitrogen ${ }^{\mathrm{TM}}$, Grand Island, USA) containing $10 \%$ fetal calf serum (Invitrogen ${ }^{\mathrm{TM}}$, Grand Island, USA), 100 units $/ \mathrm{mL}$ penicillin, $100 \mu \mathrm{g} / \mathrm{mL}$ streptomycin, and $0.2 \%$ collagenase 1A (Sigma Chemical Co, Saint Louis, USA) in a shaking bath at $37^{\circ} \mathrm{C}$. Three hours after enzymatic treatment, the muscles were dissociated into single muscle cells using Pasteur pipettes with different tip sizes.

\section{Electrophysiological measurement}

FDB muscle cells were voltage-clamped using a whole-cell patch-clamp technique. All experiments were performed at room temperature $\left(20-24^{\circ} \mathrm{C}\right)$. Patch pipettes were pulled from borosilicate glass
$\mathrm{NaOH}$. The cells were voltage-clamped at $-80 \mathrm{mV}$ in a whole-cell configuration after obtaining $G \Omega$ seals. Sixty to eighty percent of the series resistance was compensated. Currents were recorded with an EPC10 amplifier (HEKA Elektronik, Lambrecht/Pfalz, Germany) and PatchMaster software (HEKA Elektronik, Lambrecht/Pfalz, Germany), sampled at $10 \mathrm{kHz}$, and stored on a computer.

Acetylcholine (Ach) and atropine sulfate were purchased from Sigma (Saint Louis, USA). Muscle relaxants were obtained from preparations for clinical use: rocuronium (NV Organon, Oss, the Netherlands). All drugs were dissolved in the external solutions and applied by a gravity-driven perfusion system. Solutions and their dilutions to the experimental concentrations were prepared immediately before the experiments.

Test solution applications containing either ACh alone or in combination with various concentrations of rocuronium were applied for 10 seconds to the skeletal muscle cells, and the peak current was determined. To determine the effect of rocuronium on the ACh-elicited current, the solution containing the rocuronium perfused the skeletal muscle cells for three minutes prior to the application of ACh in the presence of the rocuronium. The washout time between each drug application was at least 60 seconds in order to minimize the amount of desensitization throughout the course of an experiment. Currents were acquired from five skeletal muscle cells taken from at least two mice. The control current to ACh alone was repeated after washout of the rocuronium.

Taking the mean value of these two ACh applications as the average control current, the antagonist response was calculated using the following equation:

$$
\% \text { inhibition }=100 \times\left(1-\frac{\text { current in presence of antagonist }}{\text { average control current }}\right)
$$

using a Flaming Brown micropipette puller (P97, Sutter Instrument Co, Novato, USA), ranging from one to two $M \Omega$. The pipette electrode was filled with the following solution (mM): $140 \mathrm{KCl}, 10$ HEPES, 10 EGTA, $1 \mathrm{CaCl}_{2}$, and $1 \mathrm{MgCl}_{2}$, and the $\mathrm{pH}$ was adjusted to 7.2 with $\mathrm{KOH}$. The external solution contained (mM): $5 \mathrm{KCl}, 140 \mathrm{NaCl}, 1 \mathrm{CaCl}_{2}, 1.25$ $\mathrm{MgCl}_{2}, 10$ HEPES, and 10 glucose, with $0.5 \mu \mathrm{M}$ atropine sulfate, and $\mathrm{pH}$ was adjusted to 7.4 with

\section{Statistical analysis}

Data analysis was performed off-line using Origin 8 (OriginLab, Northampton, USA) and GraphPad Prism 4 (Graphpad software Inc, San Diego, USA). Concentration-response curves were fitted to the fourparameter logistic equation by nonlinear regression analysis, and the half-maximal response $\left(\mathrm{IC}_{50}\right.$ ) values were determined. Statistical significance was assessed with one-way analysis of variance followed by Tukey's 
test. A p-value of less than 0.05 was considered significant.

\section{Results}

FDB muscle cells were chosen for this experiment due to their relatively small size for skeletal muscle cells, allowing for a better space clamp. The mean
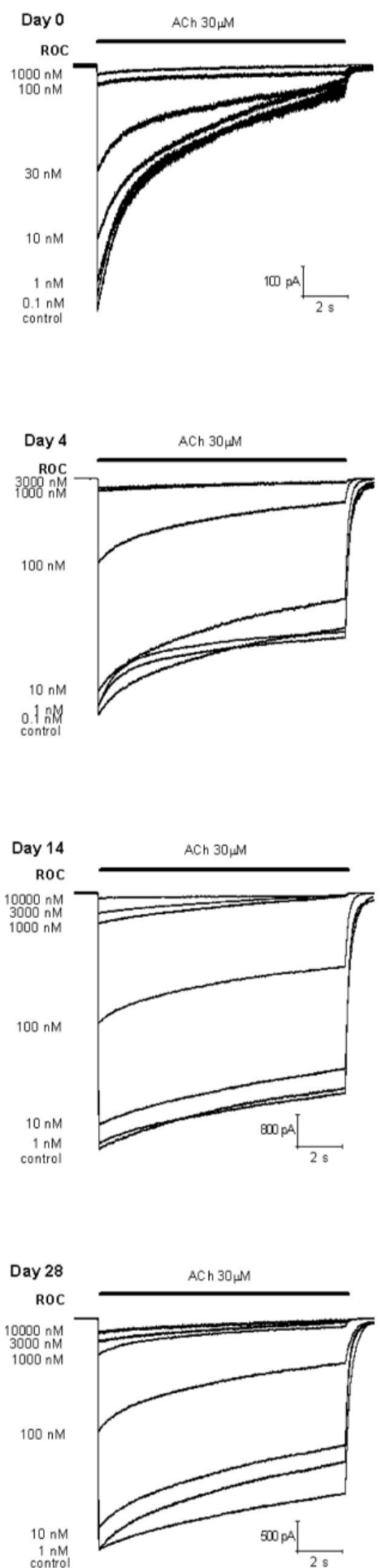

length and width of the muscle cells dissociated from innervated muscles were $387.5 \pm 9.2$ and $15.9 \pm 0.7 \mu \mathrm{m}$ $(n=10)$, respectively. Denervation had minimal effect on muscle cell size $(405.3 \pm 12.4$ and $13.5 \pm 0.6 \mu \mathrm{m}$, respectively; $\mathrm{n}=10$ ), with the small reduction in cells width being significant $(\mathrm{p}<0.05)$.
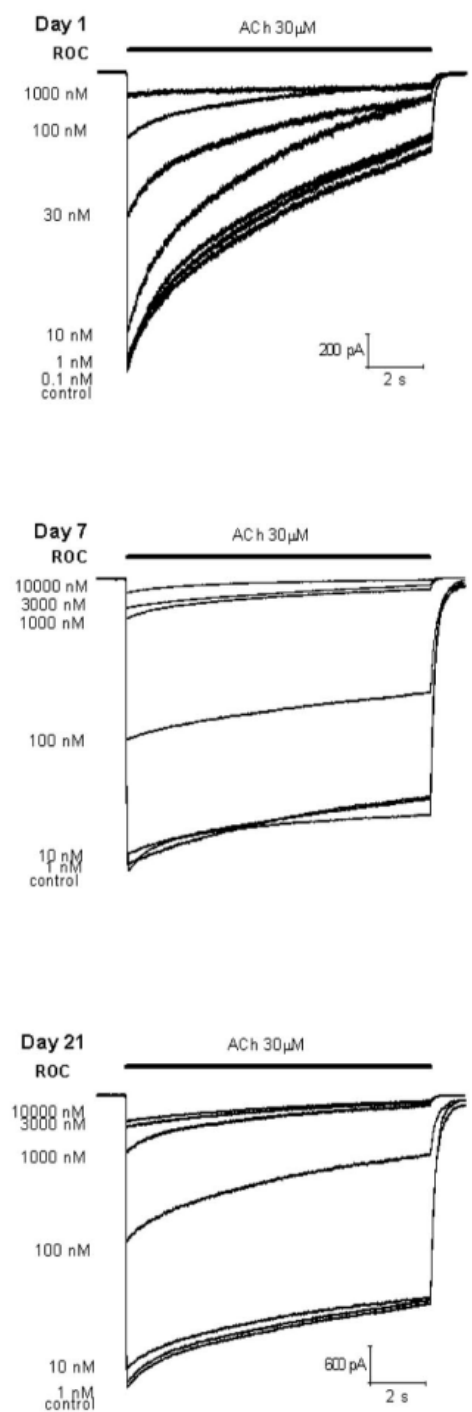

Figure 1. Concentration-dependent effects of rocuronium (ROC) on nicotinic acetylcholine receptors (nAChRs) in skeletal muscle cells at Day 0, 1, 4, 7, 14, 21, and 28 after denervation 
A concentration of $30 \mu \mathrm{M}$ ACh was used to activate $\mathrm{nAChR}$ because this concentration was close to the $\mathrm{EC}_{50}(50 \%$ effective concentration) and smaller than the estimated transient peak concentrations within the neuromuscular junction [8], but it elicited sufficient current responses without significant desensitization after repetitive application.

We examined the effects of denervation on
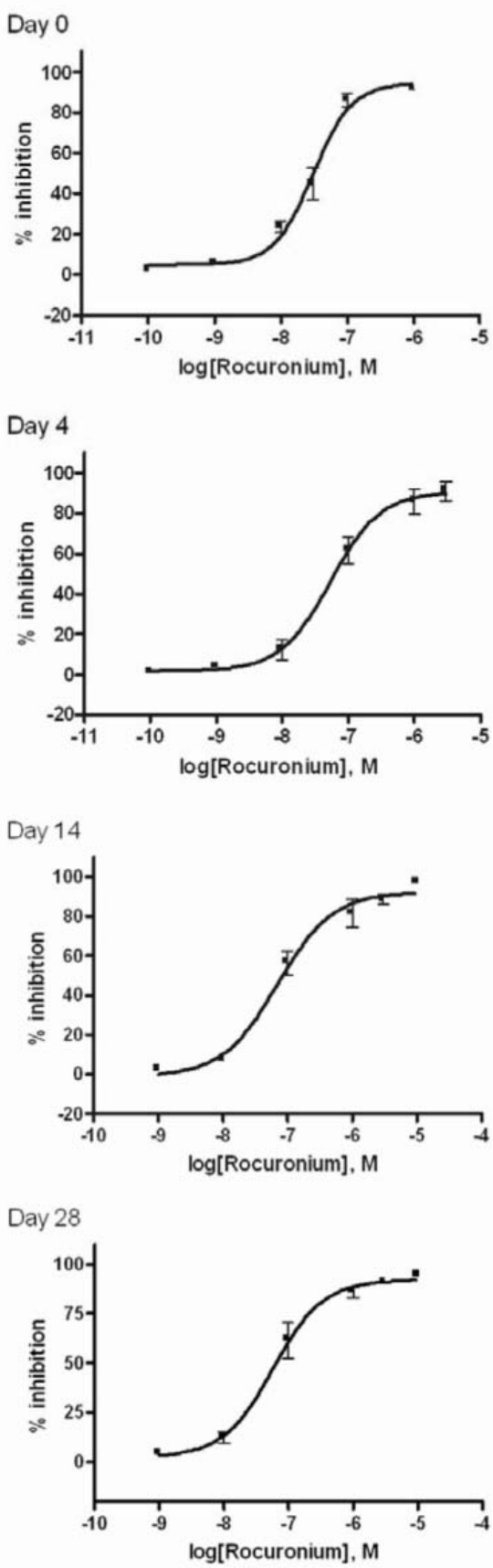

potency of rocuronium by applying ACh to innervated (or denervated) skeletal muscle. Figures $\mathbf{1}$ and $\mathbf{2}$ show effects of rocuronium on nAChRs and AChinduced currents in skeletal muscle cells after denervation. Interestingly, rocuronium reversibly inhibited ACh-elicited inward currents in a concentration-dependent manner at day $0,1,4,7,14$, 21, and 28 after denervation.

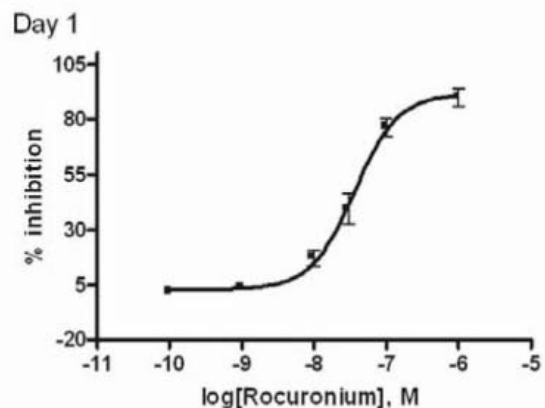

Day 7
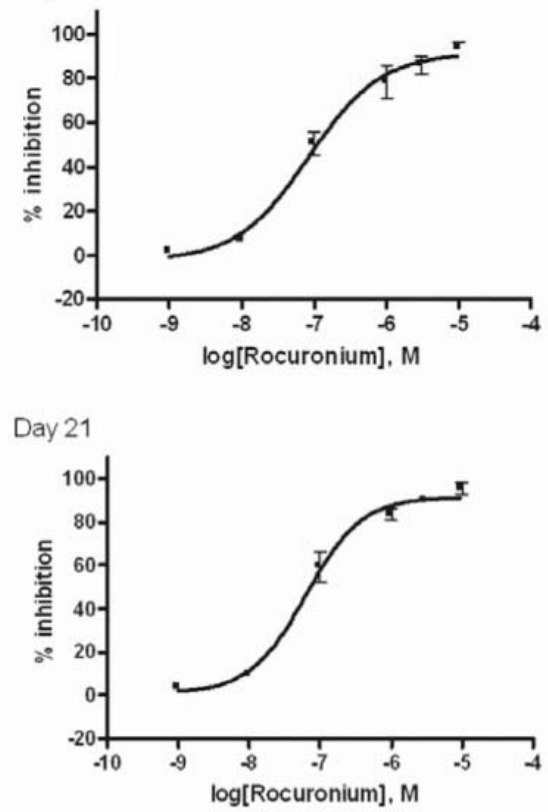

Figure 2. Concentration-response effects of rocuronium, looking at inhibition of acetylcholine-induced currents in skeletal muscle cells at Day 0, 1, 4, 7, 14, 21, and 28 after denervation 
Compared with day 0 , the IC $_{50}$ values of rocuronium were 1.2- ( $\mathrm{p}>0.05), 1.8-, 2.8-, 2.3-$, 2.1-, and 1.9-fold ( $<<0.01)$ at day $1,4,7,14,21$, and 28 after denervation (Figure 3 ).

\section{Discussion}

Determination of the changed potencies of rocuronium at nAChRs in the short-term after denervation of skeletal muscle is most important because the amount of membrane nAChRs and the proportion of -AChR after denervation change with time, which may lead to increased dosing requirements of rocuronium to complete rapid tracheal intubation.

Developing mouse denervation model according to previous reports $[8,9]$, we successfully acutely isolated FDB muscle cells of mouse. We took an ACh concentration of $30 \quad \mathrm{M}$ as the agonist concentration. This concentration was close to the $\mathrm{EC}_{50}$ concentration for the innervated control, ensured robust baseline responses, and minimized receptor desensitization due to repetitive ACh application. We used an agonist concentration of $30 \mu \mathrm{M}$ ACh used throughout the study. Although this level may be smaller than the estimated peak concentrations within the neuromuscular junction, the present experiments might be more physiologically meaningful than pure binding assays using desensitized forms of the nAChR, because the desensitized forms of the nAChRs showed different affinities for modulating agents compared with functional receptors [10-12]. We applied a holding voltage of $-80 \mathrm{mV}$ because the antagonistic effects of NDMRs are independent of holding voltages ranging from -100 to $-40 \mathrm{mV}$ $[13,14]$.
Consistent with previous reports $[6,15]$, the $\mathrm{IC}_{50}$ values of rocuronium increased after denervation, indicating that the potency of rocuronium decreased after skeletal muscle denervation. Potential mechanisms for this decreased potency of rocuronium are that denervation causes up-regulation of mature and immature $\mathrm{nAChR}$ on the muscle membrane, which would increase the amount of NDMRs required to competitively block Ach [16].

Regarding any change in nAChR numbers at day 1 , some reports have been conflicting. Ibebunjo and Martyn $[6,17]$ found no changes in nAChR numbers at day 1 after burn. However, Tsay and Schmidt [18] reported a sharp increase in the transcriptional activity of AChR subunit genes that began after approximately half a day in denervated chick skeletal muscle. In our study, although the $\mathrm{IC}_{50}$ values of rocuronium increased at day 1 , the $\mathrm{IC}_{50}$ value of rocuronium was not statistically significantly different compared to that at day 0 after denervation. Potency of rocuronium was significantly poor at day 4 , poorest at day 7 , and began to increase by day 14 . These findings are paralleled by changes in nAChRs and -AChR at the same days after denervation, as shown by other researches $[4,6$, 17]. Although the $\mathrm{IC}_{50}$ values of rocuronium further decreased after day $14, \mathrm{IC}_{50}$ values at day 28 remained significantly higher than the innervated control, which indicated that potency of rocuronium remained significantly poor at day 28 compared to that at day 0 . Consistent with our results, Adams et al. [7] observed an increase in nAChR RNA level during the first month of denervation and the level began to return to the innervated level beyond one month after denervation. However, Ma et al. [4] found that the

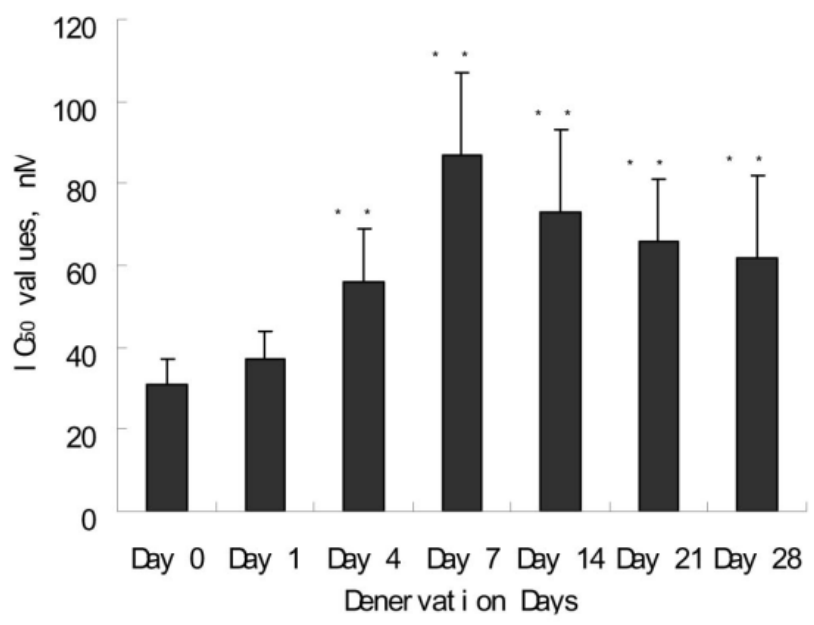

Figure 3. $\mathrm{IC}_{50}$ values of rocuronium at day $0,1,4,7,14,21$, and 28 after denervation. ${ }^{* *} \mathrm{p}<0.01$ vs. day 0 
expression of most of the nAChR subunits returned to the control level within one month after denervation.

In conclusion, short-term muscle denervation lead to a changing potency of rocuronium that might be attributed to up-regulation of mature and immature nAChR on the muscle membrane. After denervation, potency of rocuronium decreased at day 4 , decreased to bottom at day 7 , began to increase at day 14 , and remained to decrease at day 28 compared to that at day 0 . Our findings suggest that the dose requirement of rocuronium after denervation to accomplish rapid tracheal intubation be altered within one month after denervation.

\section{Acknowledgements}

This work was supported by the National Natural Science Foundation of China (No. 30571796). We thank Dr Xin-qiu Liu (Department of Neurobiology, School of Medicine, Shanghai Jiao Tong University) for his technical assistance and strong support. The authors have no conflict of interest to declare.

\section{References}

1. Hogue CW Jr, Itani MS, Martyn JA. Resistance to d-tubocurarine in lower motor neuron injury is related to increased acetylcholine receptors at the neuromuscular junction. Anesthesiology. 1990; 73: 703-9.

2. Ibebunjo C, Martyn JA. Thermal injury induces greater resistance to d-Tubocurarine in local rather than in distant muscles in the rat. Anesth Analg . 2000; 91:1243-9.

3. Gu Y, Hall ZW. Characterization of acetylcholine receptor subunits in developing and in denervated mammalian muscle. J Biol Chem. 1988; 263:12878-85.

4. Ma J, Shen J, Garrett JP, Lee CA, Li Z, Elsaidi GA, Ritting A, et al. Gene expression of myogenic regulatory factors, nicotinic acetylcholine receptor subunits, and GAP-43 in skeletal muscle following denervation in a rat model. J Orthop Res. 2007; 25: 1498-505.

5. Nosek MT, Martyn JA. $\mathrm{Na}^{+}$channel and acetylcholine receptor changes in muscle at sites distant from burns do not simulate denervation. J Appl Physiol. 1997; 82: 1333-9.

6. Ibebunjo C, Martyn JA. Thermal injury induces greater resistance to d-Tubocurarine in local rather than in distant muscles in the rat. Anesth Analg. 2000; 91:1243-9.

7. Adams L, Carlson BM, Henderson L, Goldman D. Adaptation of nicotinic acetylcholine receptor, myogenin, and MRF4 gene expression to long-term muscle denervation. J Cell Biol. 1995; 131:1341-9.

8. Wagatsuma A, Osawa T. Time course of changes in angiogenesis-related factors in denervated muscle. Acta Physiol (Oxf) 2006; 187: 503-9.

9. Magnusson C, Hogklint L, Libelius R, Tagerud S. Expression of mRNA for plasminogen activators and protease nexin-1 in innervated and denervated mouse skeletal muscle. J Neurosci Res 2001; 66: 457-63

10. Kuffler SW, Yoshikami D. The number of transmitter molecules in a quantum: an estimate from iontophoretic application of acetylcholine at the neuromuscular synapse. J Physiol. 1975; 251:465-82.

11. Paul M, Kindler CH, Fokt RM, Dresser MJ, Dipp NC, Yost CS. The potency of new muscle relaxants on recombinant muscle-type acetylcholine receptors. Anesth Analg. 2002; 94:597-603.

12. Sine SM, Claudio T. Gamma- and delta-subunits regulate the affinity and the cooperativity of ligand binding to the acetylcholine receptor. J Biol Chem. 1991; 266:19369-77.

13. Garland CM, Foreman RC, Chad JE, Hold-Dye L, Walker RJ. The actions of muscle relaxants at nicotinic acetylcholine receptor isoforms. Eur J Pharmacol. 1988; 357:83-92.

14. Fletcher GH, Steinbach JH. Ability of nondepolarizing neuromuscular blocking drugs to act as partial agonists at fetal and adult mouse muscle nicotinic receptor. Mol Pharmacol. 1996; 49:938-47.

15. Han T, Kim H, Bae J, Kim K, Martyn JA. Neuromuscular pharmacodynamics of rocuronium in patients with major burns. Anesth Analg. 2004; 99:386-92.

16. Martyn JA, White DA, Gronert GA, Jaffe RS, Ward JM. Up-and-down regulation of skeletal muscle acetylcholine receptors: effects on neuromuscular blockers. Anesthesiology. 1992; 76:822-43.

17. Ibebunjo C, Martyn J. Disparate dysfunction of skeletal muscles located near and distant from burn site in the rat. Muscle Nerve. 2001; 24:1283-94.

18. Tsay HJ, Schmidt J. Skeletal muscle denervation activates acetylcholine receptor genes. J Cell Biol. 1989; 108:1523-6. 